\title{
The use of plastic tubing in roller pumps
}

\author{
M. P. WRIGHT AND G. C. SPENCER \\ From the Nuffield Department of Surgery, Radcliffe Infirmary, Oxford, and \\ Southampton University, Southampton
}

SYNOPSIS If plastic tubing is to be used in roller pumps for propelling blood in extracorporeal circulations under occlusive conditions spring loading the rollers and accurately setting the spring force is essential. Various types and sizes of plastic tubing are compared and their performance estimated; rubber tubing used for the same purpose has been tested. The optimum spring force has been determined both for plastic and for rubber tubing.

It has recently become clear that roller pumps used for propelling blood in extracorporeal circulations may be made completely occlusive without a prohibitive rise in the rate of haemolysis. This is desirable, since the output of the pump, then becomes independent of peripheral resistance. If thick-walled Latex tubing is used in the pump setting up the roller spacing to give complete ccclusion is relatively simple, the pliable properties of rubber providing ample tolerance. But if plastic tubing is taken directly through the pump the adjustment to give complete occlusion is difficult, if not impossible. There is almost no tolerance between a position which fails to occlude and one which will split the tubing. A number of roller pumps have been designed to avoid this difficulty by spring-loading their rollers. But many of these are almost equally difficult to set up to give complete occlusion, and there have been some accidents with split tubing.

This paper describes the performance of a springloaded roller pump which was designed to avoid this difficulty, and to explore the behaviour of plastic tubing in roller pumps.

\section{DESIGN OF THE PUMP HEAD}

Early in these experiments, a possible reason was found for the difficulty in setting up the known spring-loaded roller pumps. The spring force required to occlude a Tygon tube with walls $1 / 16$ in. thick was surprisingly high, $20 \mathrm{lb}$. Very few of the pumps seen have springs strong enough to exert this force so that when they are set for occlusion, the springs are hard on their end stops. The present pump head was therefore made to allow accurate setting of the spring force up to $35 \mathrm{lb}$.

The roller head was made to fit an existing Mark pump, which has an outer horseshoe 6 in. in diameter, extending over half a circle. Three rollers were therefore used to make sure of occlusion in all positions. The mechanical arrangement is shown in Fig. 1.

The springs are $1 \frac{1}{2}$ in. long, and $\frac{1}{2}$ in. in internal diameter, being made of eight turns of 15 gauge wire. The movement of the roller arms is limited by end stops. These are set up to take the weight of the spring just as it begins to ride off the horseshoe with the tubing in place. The spring force is set up with a spring balance hooked round a pin placed in the roller axle. With the line of the balance passing through the centre of the pump head, the spring is adjusted until the required pull will just lift the roller from its stop.

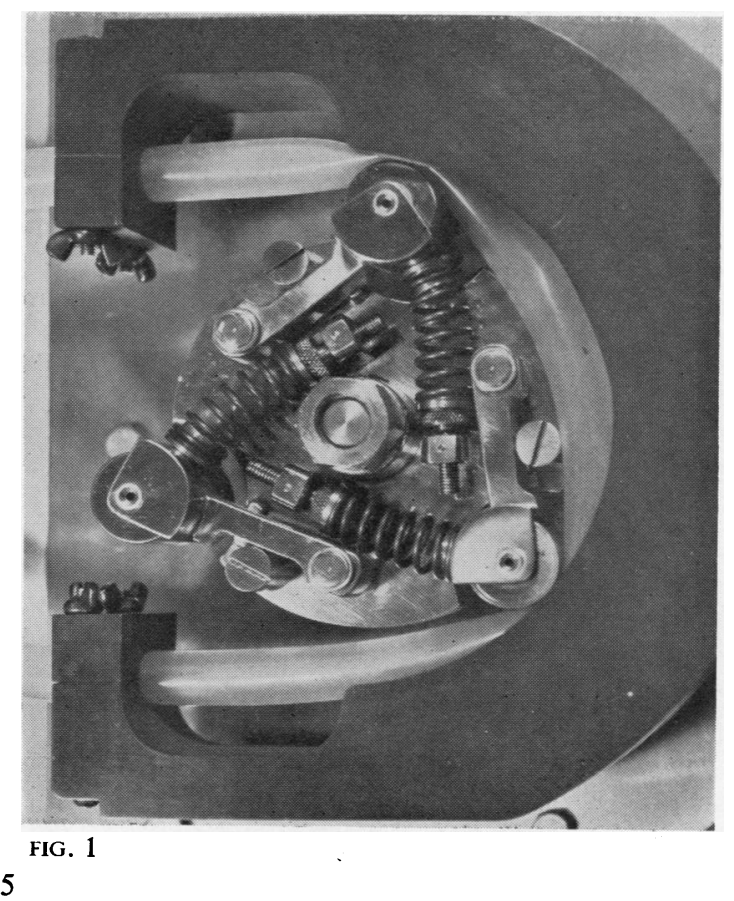




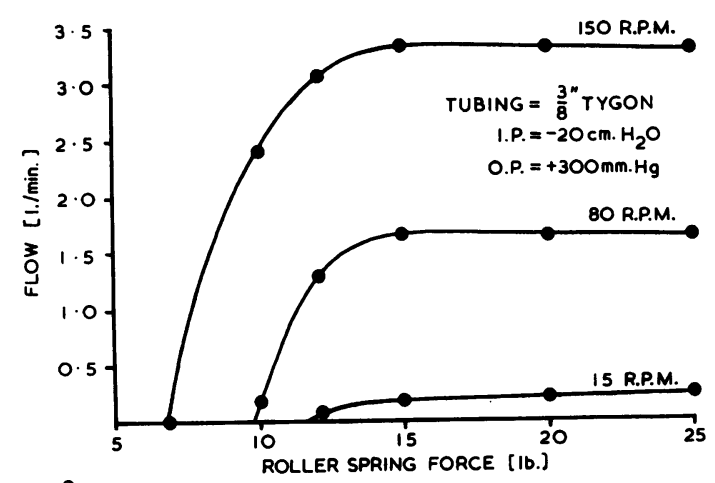

FIG. 2

\section{PERFORMANCE OF THE PUMP}

COMPLETE OCCLUSION If occlusion is incomplete the output of the pump is reduced when it works against a head of pressure. The effect is more noticeable at low rates of revolution, since the rate of regurgitation represents a greater proportion of the total pump output. Fig. 2 shows how the output of the pump varies with spring force, at three different speeds. The tubing used was Tygon, $3 / 8$ in. I.D., with $1 / 16$ in. wall. All three curves are seen to become flat at tensions above $15 \mathrm{lb}$., when complete occlusion is reached.

Other types of tubing gave similar families of curves, indicating accurately the force required to give complete occlusion of each. With plastic tubing, the occlusive force was very similar for Tygon and for Clearclave. It varied little with the diameter of the tube but more with the wall thickness. Clearclave tubing $\frac{1}{2}$ in. I.D. with $3 / 32$ in. wall required a minimum of $20 \mathrm{lb}$.

When Latex tubing $5 / 16$ in. I.D., with $1 / 8$ in. wall, was used, $20 \mathrm{lb}$. tended to drag the tube round with the rollers. The minimum occlusive force was $10 \mathrm{lb}$. and $12 \mathrm{lb}$. which gave satisfactory operation without the necessity for a flange on the tube to keep it in position.

SPLITTING OF PLASTIC TUBES Plastic tubes with 1/16 in. walls tended to split, especially at low temperatures, if spring tensions above $30 \mathrm{lb}$. were used. But no splitting was seen at a tension of $20 \mathrm{lb}$., even after many hours at $5^{\circ} \mathrm{C}$. Tubing with $3 / 32$ in. walls split on one occasion after a short period at $5^{\circ} \mathrm{C}$. with $25 \mathrm{lb}$. pressure but did not split at $20 \mathrm{lb}$.

PUMP OuTPuT This was found to vary with pressure and with temperature.

Variation with output pressure With complete occlusion, the output for any one tube is independent of output pressure, and will give a linear calibration against speed at least from 5 to 200 r.p.m. Output variations have been found for different tubes of the same nominal size up to about $\pm 12 \%$, presumably due to differences in dimensions.

Variations with input pressure and with temperature When regurgitation is prevented, output under any given conditions is controlled by the passive filling of the tube $\stackrel{0}{=}$ behind the roller. This is caused by the tendency of the 음 tube to reassume its normal round shape, i.e., its elasticity, and by the input pressure. Any tubing worth using must $\stackrel{\vec{S}}{\rightarrow}$ have sufficient elasticity to fill against an appreciable $\bar{C}$ negative pressure, and ideally, the degree of filling should $\frac{\bar{\sigma}}{\sigma}$ not vary over the range of pressures normally encountered, $\overline{\bar{\omega}}$ e.g., +10 to $-30 \mathrm{~cm}$. of water. With Latex tubing, $\overrightarrow{\mathbb{D}}$ $5 / 16 \mathrm{in}$. diameter and 1/8 in. wall, filling is unaffected by $\varrho$ input pressures between +10 and $-60 \mathrm{~cm}$. of water, or by temperatures between 5 and $40^{\circ} \mathrm{C}$. But with plastic $\vec{O}$ tubing, the elasticity is barely adequate, and is considerably affected by reduction in temperature; the plastic becomes stiffer but less elastic.

Figure 3 shows the outputs for three different tubes, $\frac{\mathrm{O}}{\mathrm{g}}$ all at the same speed and output pressure. The curves? show the change in output with temperature between $\vec{c}$ 5 and $40^{\circ} \mathrm{C}$. There are two curves for each tube, one at $\omega$ +10 and the other at $-60 \mathrm{~cm}$. $\mathrm{H}_{2} \mathrm{O}$ input pressure. $\mathrm{N}$ The Latex tubing shows no change, either with tempera-

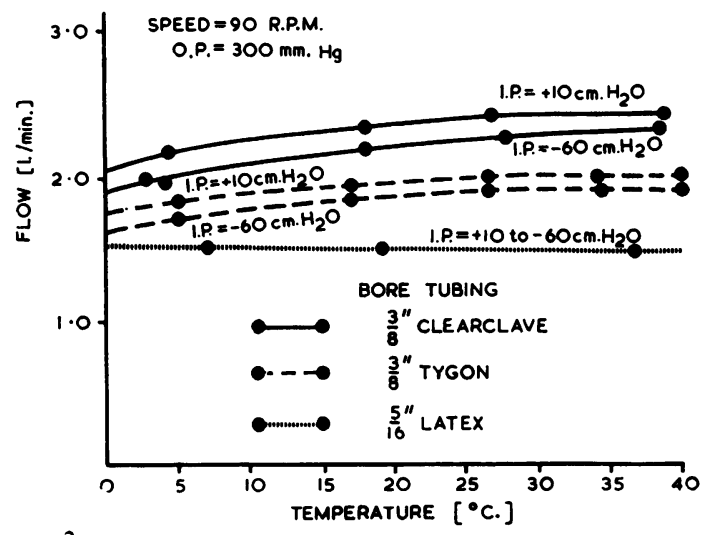

FIG. 3

ture or with input pressure. The two plastic tubes are both of nominal $3 / 8$ in. internal diameter, with $1 / 16$ in. wall thickness. One is Tygon and the other is Clearclave. The overall difference in output between the two is due to difference in their actual internal diameter.

The Tygon tubing shows a $4 \%$ drop in output due to $D$ negative input pressure and a $10 \%$ drop when the temperature is lowered from $40^{\circ} \mathrm{C}$. to $5^{\circ} \mathrm{C}$. The Clearclave tube showed corresponding drops of $5 \%$ and $12 \%$.

Figure 4 shows two pairs of curves for $\frac{1}{2}$ in. plastic $N$ tubing. One has a 1/16 in. wall and is Tygon; the other $N$ has a 3/32 in. wall and is Clearclave. It is obvious that the $\omega$ elasticity of the thinner-walled tube is inadequate. It shows a drop in output of $15 \%$ with negative input 0 pressure, and $30 \%$ with fall in temperature. The thicker- $\mathbb{D}$ walled tube still shows $17 \%$ drop at $5^{\circ} \mathrm{C}$. but the drop with negative input pressure is only $2 \%$.

\section{RATE OF HAEMOLYSIS}

The rate of haemolysis due to the pump was measured by $\varrho$ circulating 1 pint of fresh citrated blood into and out of a (1) 


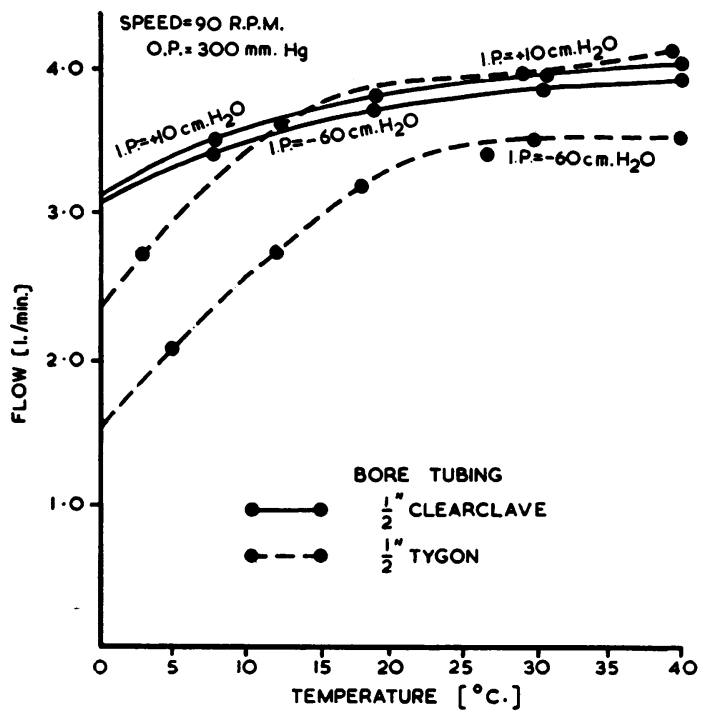

FIG. 4

small reservoir at 3 litres per minute for two hours. New half-inch Tygon tubing was used, and the spring tension was $20 \mathrm{lb}$. Samples were taken at intervals. Haemolysis was measured by diluting the plasmas one in four with water, and reading their density in a normal haemoglobin photoelectric photometer, setting the zero with the initial sample.

The rate of haemolysis was then calculated in $\mathrm{mg}$. \% $\mathrm{Hb}$ per transit of the whole volume through the pump. The rate of haemolysis for the first half hour, or 150 transits, was very low, being $6 \mathrm{mg}$. $\%$, or $0.04 \mathrm{mg}$. $\%$ per transit. After this, the rate became steady with time at $0.123 \mathrm{mg}$. \% per transit.

\section{DISCUSSION}

NUMBER OF ROLLERS For minimum haemolysis, there should be as few rollers as possible, provided that one is in contact all the time. One roller may be used, as in the new Mayo pump, if it is in contact for more than $360^{\circ}$. For simplicity of manufacture, perhaps two rollers and a 5/8 circle horseshoe are best. Three rollers are used in this pump only because the horseshoe was half a circle.

Reduction of the number of rollers has a further theoretical advantage: the resulting pulse pressure is greater and the pulse rate closer to physiological rates. The intermittent nature of the normal arterial pressure is probably of importance in maintaining the normal circulation of the tissue fluid in and out of the capillaries and into the lymphatics.

TYPE AND DIMENSIONS OF TUBING It is obvious from these tests that Latex tubing will give the most reliable calibration of pump output, and is not subject to splitting. But if plastic tubing is indicated for the sake of simplicity, then care must be taken in choosing its diameter and wall thickness. Halfinch diameter tube with $1 / 16$ in. walls is very commonly used but it is obviously quite unsuitable in deep hypothermia, where flow rates should be known. Increasing the wall thickness to 3/32 in. improves the output characteristics, but leads to a very small margin between the tension required for occlusion and that which may split the tube at low temperatures.

Three-eighth inch tubing with 1/16 in. walls was most generally satisfactory, in that its performance was acceptable and its safety margin between occlusion and splitting was generous. With it we have used a spring force of $20 \mathrm{lb}$. to allow for change in the spring setting during operation and variation in tube thickness. One $3 / 8$ in. tube will give outputs up to $41 . / \mathrm{min}$. at 180 r.p.m. If greater flows are required, two tubes may be put in parallel but if this complication has to be faced one might as well use Latex tubing.

HAEMOLySiS Hall, James, Lucas, and Waterston (1958) have pointed out that the calculation of haemolysis rate in terms of $\mathrm{mg}$. \% plasma haemoglobin per transit of the pump allows direct comparison of one experiment with others using different total volumes and flow rates. Our figure of 0.123 mg. \% per transit is lower than the best of the pumps they tested. It also compares well with the figures calculated from other published results, e.g., Leonards and Ankeney's (1958) occlusive roller pump gave $0.22 \mathrm{mg}$. \% per transit, and Cahill (1959), comparing a number of different pumps, gave levels corresponding to $0.133 \mathrm{mg}$. \% per transit as a low level and $0.79 \mathrm{mg}$. \% per transit as a high level.

\section{REFERENCES}

Cahill, J. J. (1959). Trans. Amer. Soc. for Artificial Internal Organs $5,224$.

Hall, J. E., James, P. A., Lucas, B. G. B., and Waterston, J. D. (1958). Thorax, 13, 34.

Leonards, J. R., and Ankeney, J. L. (1958). Trans. Amer. Soc. for Artificial Internal Organs, 4, 69. 